\title{
Design and optimized analysis of high speed precise machine tool spindles
}

\author{
Jeevan Raju B ${ }^{1}$, Rama Kotaiah $K^{2}$, Jakeer Hussain Shaik ${ }^{3}$ \\ ${ }^{1}$ Research Scholar, Dept. of Mechanical Engineering, KLEF University, Vaddeswaram, India. \\ ${ }^{2}$ Professor, Dept. of Mechanical Engineering, KITS Guntur, India. \\ $3^{3 *}$ Associate Professor, Dept. of Mechanical Engineering, Malla Reddy Engineering College, Telangana, India. \\ 3jakeershaik786@gmail.com
}

Article History: Received: 11 January 2021; Accepted: 27 February 2021; Published online: 5 April 2021

\begin{abstract}
Upcoming machine tools need to be extremely efficient systems to maintain the needed intellectual performance and stability. The spindle tool system is necessary to optimize which enhances the rigidity of the spindle and in turn produces the cutting stability with higher productivity. Prediction of the dynamic behavior at spindle tool tip is therefore an important criterion for assessing the machining stability of a machine tool at design stage. In this work, a realistic dynamic high-speed spindle /milling tool holder/ tool system model is elaborated on the basis of rotor dynamics predictions. The integrated spindle tool system in analyzed with the Timoshenko beam theory by including the effects of shear and rotary deformation effects. Using the frequency response at the tool tip the corresponding stability lobe diagrams are plotted for the vertical end mill system. Furthermore an optimization study is carried out at design stage for the bearing system and the rotor positions for maximizing the chatter vibration free cutting operation at the desired depth of cuts with precise rotational speeds.It is markedly found that the first mode of vibration had a large impact on the dynamic stability of the system. The parametric studies are conducted such as tool overhang and bearing span and the influence of these on the system dynamics are identified and the corresponding stability lobe diagrams are plotted. It is evidently found that the second mode of the frequency response has critically affected the bearing span and competing lobes are identified. These results are assisted to design a spindle bearing system at the desired machining conditions. A neural network based observer is designed based on the simulation resultsto predict optimum design parameter values.

Keywords: Frequency response; spindle design; milling stability; lobe diagrams; parametric studies.
\end{abstract}

\section{Introduction}

In recent years, the technical advancement of high speed machining (HSM) has been significant. Improved spindle designs make speeds for micro-milling up to 20,000 rpm or higher. The machining at higher axial depths of cuts and spindles will achieve high material removal speeds (MRR). However, it creates complex volatility in material removal speeds, poor surface finish and probably destroy the machinery and the parts. However, it is not feasible to process at higher axial depths of cutting. In particular the establishment of reliable cutting force and chatter models is important to achieve a highly efficient production inspired by the current requirements of highly automated production, low-cost and high quality finishing. Analytically, the optimal depth cutting diagram combination of the stability lobe separates stable cutting conditions from unstable conditions and is represented in conjunction with the axial depth of the cut and spindle speed in rpm. The models used to predict the stability lobes need the fundamental frequencies, the geometry of the instrument, cutting parameters and cutting force coefficients. Chatter vibrations thus remain the key limiting factor in increasing mechanical machine tool metal removal speeds.

Chatter may occur at unique axial depth-of-cut and spindle speed configurations throughout the milling process. Several experiments on regenerative talk were undertaken since the late 1950s, such as Altintas and Budak[1], which used an alternative technique in order to turn the complex milling equations into invariant but radial immersion systems. Times are approximated by their components in the Fourier series and the chatter free axial depth of the cuts are determined and spindle speeds calculated. Tanga and Song[2] have developed a new way that recognizes higher excited frequencies (i.e. passage of tooth) of device multi modes of dynamics.(i.e. tooth moving frequency) and broader spindle velocity ranges, and these high-speed milling stability constraints help pick milling parameters for the optimum rate of metal removal without chatter. The Dynamic High-Speed Spindle Bearing System was developed and revised based on rotor dynamical forecasts and on experimental modal detection by Gagnola and Bouzgarrou[3]. In addition, owing to the improved dynamic stiffness, the stability limits were determined from the rotating FRF and are considerably higher.A technique for achieving the instability or equilibrium lobes, applicable as both the machine structure and the machined workpiece have identical complex behaviors, was investigated by Bravo et al.[4]. Therefore, based on the relative movement of both processes, a three-dimensional lobe diagram has been created to cover all the intermediate stages of wall machining. By using inverse study of the self-excited chatter vibration measured during an end-milling trial, Suzuki et al.[5] proposed a novel way of defining the transfer function. To minimize errors between the analytical and experimental stability limits in the axial depth, and modal parameters were optimized. 
A design flow map was created by Lin and Tu [6] to reflect the overall problems of the spindle design and eight design parameters were defined. In order to explore their effect on the natural frequencies of the spindle mechanism, a design sensitivity analysis of these eight design parameters is then performed based on an applied finite element process model. The results of bearing preload mechanisms on the dynamic output of high speed spindles were presented by Cao et al.[7]. Finally, they demonstrated that the rigid preload system is more powerful than constant preload in preserving the dynamic stiffness of spindles at high speeds and under cutting loads.By using the conventional transfer matrix system (TMM) and the Jones-Harris rolling bearing model, including centrifugal force and gyroscopic effects, Jiang and Zheng[8] developed a coupling model of a motorized high-speed spindle assisted by angular-contact ball bearings. In order to reflect the effects of bearing clearance, Peng et al.[9] used a discontinuous stiffness model, using numerical simulation procedure, the impact of the bearing clearance on the chatter stability of the milling mechanism was studied. The chatter lobes are plotted and it shows that the clearance of the bearing will reduce the frequency of the dominant chatter and restrict the cutting axial distance.

The effect of the spindle overhang and bearing duration on the frequency response of the spindle bearing mechanism was investigated by Gao and Meng [10]. The continuous two step Timoshenko beam model specifies the transition functions at the spindle nose. The effect of bearing preload on the spindle and tool tip FRFs was presented by Ozturk et al. [11]. The relationship on the tested spindle between preload and spindle speed is given. The precision of the stability lobes was improved by Raphael et al.[12] and proved that tap tests need to be repeated at the same stage. The effect of the spindle electronic location and speed control on the precision of the SLD was investigated using a specific CNC computer machining.By finding the highest negative actual portion of the FRF, Zheng et al. [13] suggested a way to evaluate the worst spindle speeds and their critical limiting chip width for turning operation. An updated FRF model is adapted to evaluate the worst spindle speeds and the critical limiting axial depth of cut in explicit, theoretical formulas for a two degree of freedom milling method of planar isotropic dynamics. An interactive model was analyzed by Liu and Chen[14] for the complex behaviour of motorized spindles. To perform a design sensitivity analysis for the device, a design flow map is then constructed.

Several recent studies are still being carried out to predict the tool tip frequency response functions to affectively plot the stability lobe diagrams on different issues related to the design stage. The practical spindle tool unit of the vertical CNC milling machine tool is considered for analysis in the present paper. Using the Timoshenko finite element beam model, the governing differential equations of spindle system motion are used and numerical simulations are carried out. Tool-tip FRFs are calculated to efficiently plot the stability lobe diagrams using the known radial immersion depths in the high-speed end-mill machining process for this spindle bearing system. The organization of the paper is as follows: Section-2 give details of the spindle-bearing unit and finite element modelling and Section-3 discuss the dynamics and cutting forces of the machining process during the end-milling operation. In chapter-4, results and discussions are presented.

\section{Finite element analysis formulation}

By modelling the restricted spindle rotation system the dynamics of the spindle tools unit can be well determined, as seen in Fig. 1. To model the spinning spindle, a two-node beam element incorporating the principle of Nelson's Timoshenko beam [16] with rotary inertia and shear deformation effect is used. It consists of five elements, six nodes and a complete of 28 degrees freedom is considered. Two angular ball bearings supported at the fourth and fifth nodes of the shaft. The cutting tool is expected to be rigidly attached to the spindle shaft tool holder. The complete equations in matrix form can be written by applying the Hamilton principle:

$$
[M]\{\ddot{q}\}+[[C]-\Omega[G]]\{\dot{q}\}+\left([K]-\Omega^{2}\left[M_{c}\right]\right)\{q\}=F
$$

Where the mounted mass, viscous damping and stiffness matrices $[\mathrm{K}]$ are $[\mathrm{M}],[\mathrm{C}]$ and $[\mathrm{K}]$, the rotation speed is the $[G]$, whereas the word $\left[M_{C}\right]\{q\}$ implies a gyroscope matrix, the spring force's softening impact. Different ball bearings support the front and rear sections of the spindle shaft. The stiffness of angular contact covers depends on the loads applied and the layout of the covering. Several analytical formulas in the literature were suggested for measurement of the axial preload $\left(\mathrm{F}_{\mathrm{a}}\right.$, ball diameter $\left(\mathrm{D}_{\mathrm{b}}\right)$ ) axial stiffness of the rollers, ball diameters $\left(\mathrm{N}_{\mathrm{b}}\right)$, and contact angle of the static contact ball bearing $(\theta)$, and one of these were proposed [16].

$$
\mathrm{k}_{\mathrm{xx}}=\mathrm{k}_{\mathrm{yy}}=1.77236 \times 10^{7} \times\left(\mathrm{N}_{\mathrm{b}}^{2} \cdot \mathrm{D}_{\mathrm{b}}\right)^{1 / 3} \frac{\cos ^{2} \theta}{\sin ^{1 / 3} \theta} F_{a}^{1 / 3} \quad \mathrm{~N} / \mathrm{m}
$$

With respect to above stiffness at the bearings the assembled mass, stiffness and gyroscopic matrices are formulated. Real and imaginary portions of the FRF can explicitly be expressed as follows [15]:

$[\mathrm{H}(\mathrm{j} \omega)]=[\operatorname{Re}(\omega)]+\mathrm{j}[\operatorname{Im}(\omega)]=\left[-[\mathrm{M}] \omega^{2}+\mathrm{j} \omega[[\mathrm{C}]-\Omega[\mathrm{G}]]+\left([\mathrm{K}]-\Omega^{2}\left[\mathrm{M}_{\mathrm{c}}\right]\right)\right]^{-1}$ 
Re and Im are the actual and imagination components of the spindle tool tip's frequency response feature.

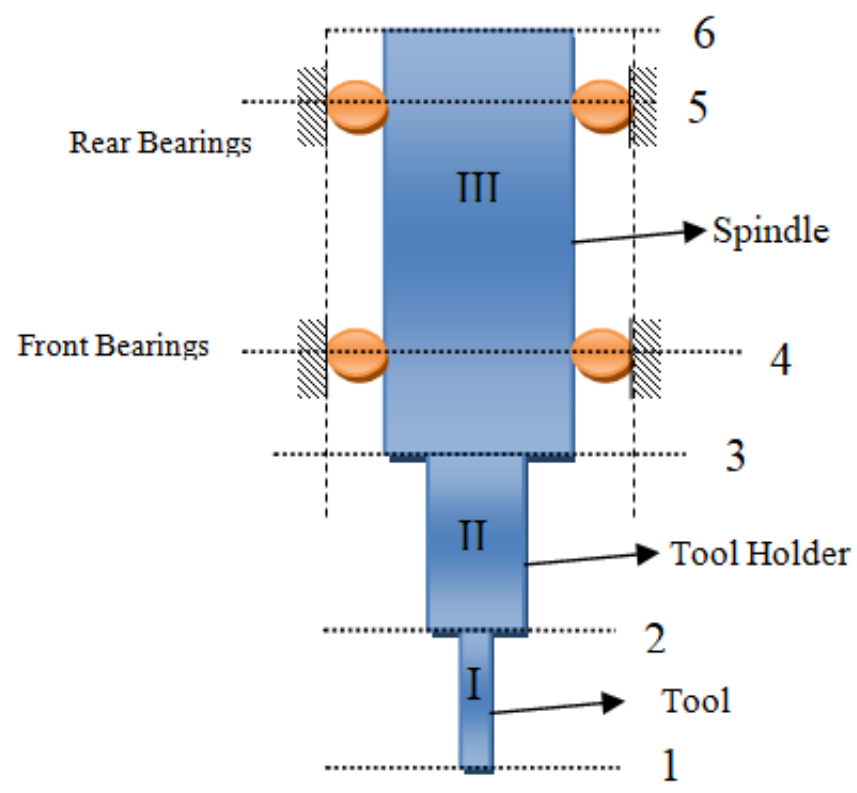

Fig.1. Equivalent model for the finite element analysis

\section{Dynamics of Cutting Process}

In the present paper, a two-dimensional cutting force model is used to represent the millingprocess to arrive at the cutting displacements in tangential and radial directions as shown in Fig 2

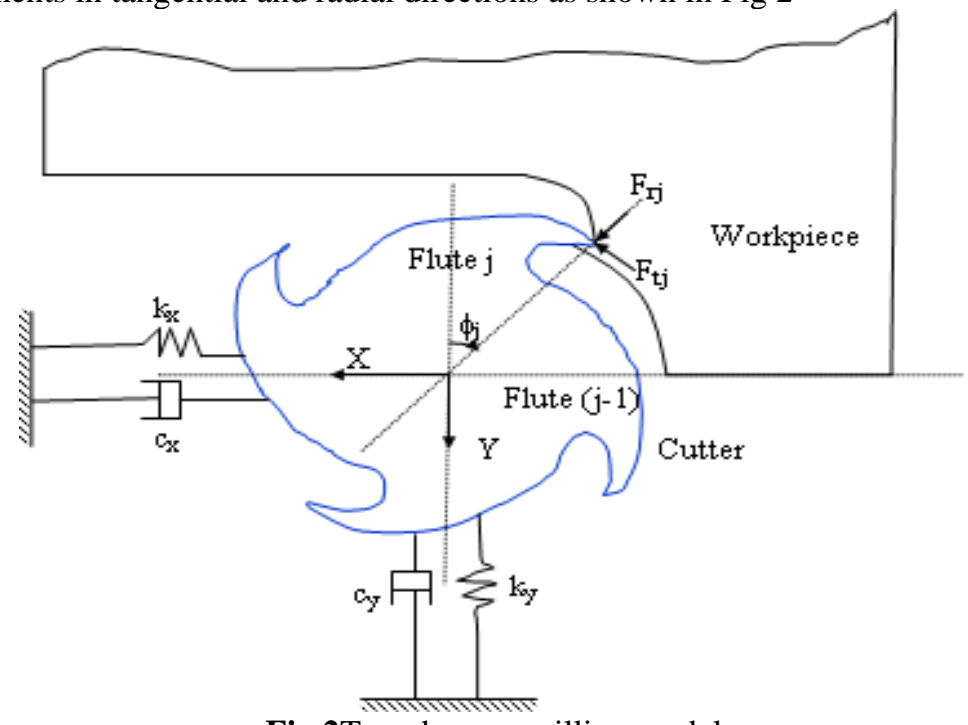

Fig.2Two degrees milling model

The angular spindle speed is taken as $\Omega$ in $\mathrm{rad} / \mathrm{s}$, and the corresponding engagement with the time period (t) is taken as $\phi_{j}(t)=\Omega t$. In the radial direction, the total variable chip thickness is taken as follows:

$$
h\left(\phi_{j}\right)=\left\{f_{t} \sin \left(\phi_{j}\right)+\delta x \sin \left(\phi_{j}\right)+\delta y \cos \left(\phi_{j}\right)\right\} * l\left(\phi_{j}\right)
$$

Here the $\delta \mathrm{x}$ and $\delta \mathrm{y}$ are the relative displacements of the present and previous tooth periods. The switching function i.e $l\left(\phi_{j}\right)$ is taken as [25]:

$$
l\left(\phi_{j}\right)=\left\{\begin{array}{cc}
1 & \text { when } \phi_{\mathrm{s}} \leq \phi_{\mathrm{j}} \leq \phi_{\mathrm{e}} \\
0 & \text { when } \phi_{\mathrm{j}}<\phi_{\mathrm{j}} \text { or } \phi_{\mathrm{j}}>\phi_{\mathrm{e}}
\end{array}\right.
$$


Where suffix ' $\mathrm{j}$ ' represents the tooth and the starting and the exit of the cutter engagement is $\phi_{s}$ and $\phi_{e}$ respectively. The cutting forces in the radial and tangential directions is represented as in terms of the chip thickness and axial depth of cut:

$$
F_{r, j}(\phi)=\cos (\beta) K_{s} b h\left(\phi_{j}\right)=K_{r} b h\left(\phi_{j}\right)(6)
$$

$$
F_{t, j}(\phi)=\sin (\beta) K_{s} b h\left(\phi_{j}\right)=K_{t} b h\left(\phi_{j}\right)
$$

Further, the coefficients of cutting force interms of specific force $(\mathrm{Ks})$ and force angle $(\beta)$ is taken as follows:

$$
K_{r}=K_{s} \times \cos (\beta)
$$

$$
K_{t}=K_{s} \times \sin (\beta)=\left[\frac{K_{s}}{\sqrt{1+K_{r}^{2}}}\right]
$$

The local cutting forces acting on the tooth ' $\mathrm{j}$ ' in the $\mathrm{x}-\mathrm{y}$ system is represented as:

$$
f_{x}=-F_{t} \cos (\phi)-F_{r} \sin (\phi)
$$

$$
f_{y}=F_{t} \sin (\phi)-F_{r} \cos (\phi)
$$

The total cutting forces are arrived over the summation from tooth ' $\mathrm{j}$ ' to ' $\mathrm{N}_{\mathrm{t}}$ ' and are expressed as follows:

$$
F_{x}(\phi)=\sum_{j=1}^{N_{t}} F_{x, j}
$$

$$
F_{y}(\phi)=\sum_{j=1}^{N_{t}} F_{y, j}
$$

These total forces are expressed in the matrix form $[\mathrm{A}(\mathrm{t})]$ and it depends on the angular position of the tool as follows:

$$
\{F\}=\left\{\begin{array}{l}
F_{x} \\
F_{y}
\end{array}\right\}=\frac{1}{2} b K_{t}[A(t)]\left\{\begin{array}{l}
\Delta x \\
\Delta y
\end{array}\right\}
$$

These expressions are periodic with the tooth pitch $\phi_{p}=\frac{2 \pi}{N_{t}}$ (rad) and tooth period $\tau=\frac{60}{\Omega N_{t}}$ (s). These time-dependent forces are converted into the Fourier domain by accounting for the mean values as:

$$
\left[A_{0}\right]=\frac{N_{t}}{2 \pi}\left[\begin{array}{ll}
\alpha_{x x} & \alpha_{x y} \\
\alpha_{y x} & \alpha_{y y}
\end{array}\right]
$$

Here the directional orientation factors is represented as:

$$
\begin{gathered}
\alpha_{x x}=\frac{1}{2}\left[\left(\cos (2 \phi)-2 K_{r} \phi+K_{r} \sin (2 \phi)\right)\right]_{\phi_{s}}^{\phi_{e}} \\
\alpha_{x y}=\frac{1}{2}\left[\left(-\sin (2 \phi)-2 \phi+K_{r} \cos (2 \phi)\right)\right]_{\phi_{s}}^{\phi_{e}} \\
\alpha_{y x}=\frac{1}{2}\left[\left(-\sin (2 \phi)+2 \phi+K_{r} \cos (2 \phi)\right)\right]_{\phi_{s}}^{\phi_{e}} \\
\alpha_{y y}=\frac{1}{2}\left[\left(-\cos (2 \phi)-2 K_{r} \phi-K_{r} \sin (2 \phi)\right)\right]_{\phi_{s}}^{\phi_{e}}
\end{gathered}
$$

These above equations are again represented in terms of oriented frequency factors and the chatter frequency as:

$$
\operatorname{det}\left([I]+\Lambda\left[H_{o}\left(i \omega_{c}\right)\right]\right)=0(20)
$$

Where $\Lambda=-\frac{N_{t}}{4 \pi} b K_{t}\left(1-e^{-i \omega_{c} \tau}\right)$

The complex eigenvalue for equation (20) is considered in terms of cutting coefficients, chatter frequency, and immersional angles. The average depth of cut for a number of teeth $(\mathrm{Nt})$ is evaluated as follows: 


$$
b_{\lim }=-\frac{2 \pi}{N_{t} K_{t}} \Lambda_{\mathrm{Re}}\left(1+\kappa^{2}\right)
$$

Where $\kappa=\frac{\Lambda_{\mathrm{Im}}}{\Lambda_{\mathrm{Re}}}=\frac{\sin \omega_{c} \tau}{1-\cos \omega_{c} \tau}$

The tooth passing period is expressed as $\tau=\frac{1}{\omega_{c}}(\varepsilon+\gamma 2 \pi)$, here $\gamma=0,1,2 \ldots$ is the number of the lobes and phase shift is $\varepsilon=\pi-2 \mu$ and $\mu=\tan ^{-1}(\kappa)$. Finally, the spindle speed interms of tooth passing period and the number of teeth is expressed as follows:

$$
\Omega=\frac{60}{N_{t} \tau}(\mathrm{rpm})
$$

After arriving at the frequency responses at the tooltip, the stability lobe diagrams are plotted between the average stable depth of cut and spindle speeds.

\section{Results with Discussions}

In order to validate the full-order FEM the following parameters employed as shown in Table-1.

\begin{tabular}{|c|c|c|c|c|c|c|}
\hline \multicolumn{7}{|c|}{ Element of the spindle } \\
\hline Parameter & $\# 1$ & $\# 2$ & \#3 & \#4 & \#5 & \\
\hline Length(m & 62 & 51 & 111 & & 90 & 47 \\
\hline $\begin{array}{l}\text { m) } \\
\text { diameter( }\end{array}$ & 12 & 40 & 120 & & 120 & 120 \\
\hline $\mathrm{E}(\mathrm{Pa})$ & $10^{11} \begin{array}{c}6.68 \times \\
\end{array}$ & $10^{11}{ }^{2.1 \times}$ & ${ }_{11} 2.1 \times 10$ & 11 & $2.1 \times 10$ & $2.1 \times 10^{11}$ \\
\hline
\end{tabular}

Table 1 Parameters of the full-order finite element model

The frequency response is evaluated at the tool tip by the considering the centrifugal effect and the dynamics of bearings at the rear and front position at spindle speed of $5000 \mathrm{rpm}$. Using MATLAB programming the responses are arrived with the mountings of the bearings calculation, the responses are shown in Figure. 3 and it is observed that the peak frequency is $2250 \mathrm{~Hz}$. Similarly, the corresponding real part and the imaginary part of the frequency responses are shown in Figure 3(b).

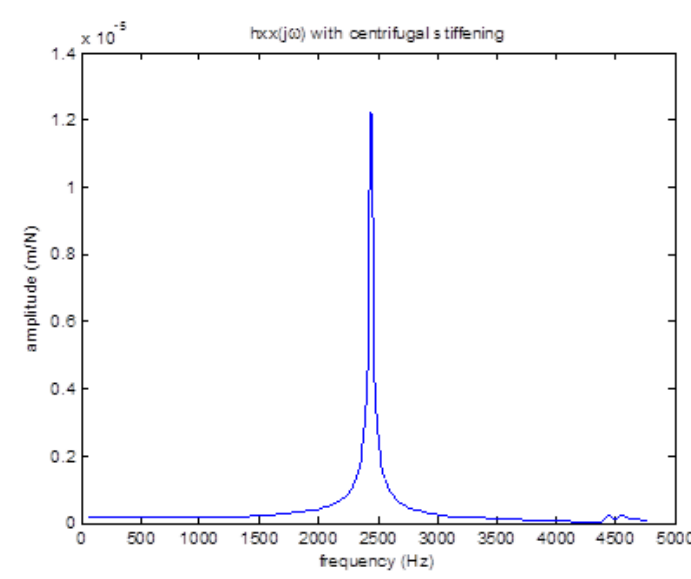

(a) Absolute tool tip FRF
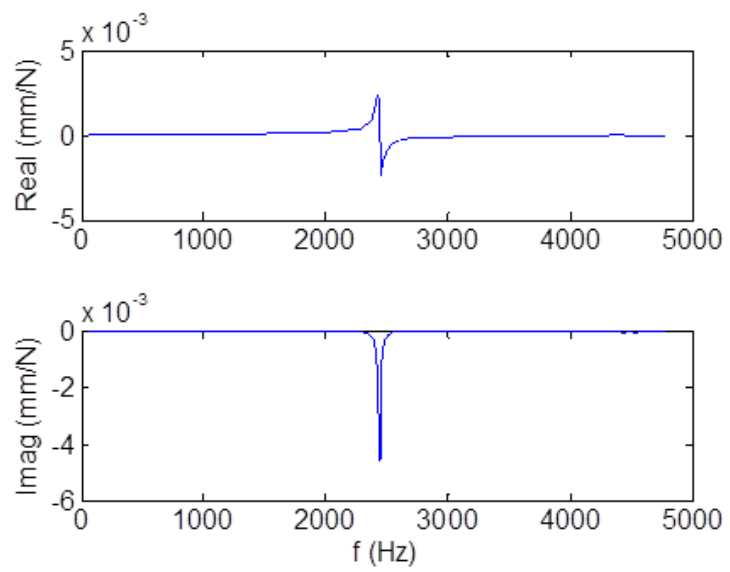

(b)Real and imaginary parts

\subsection{Prediction of stability charts for different depths of radial immersions}


The stability charts are the graphical representation between the stable and the unstable cutting zones which is represented as the functional relationship between the axial depth of cut and the spindle speed and technically it called as the stability lobe diagram. The prime most advantage of these lobe diagrams is the maximum metal removal rates can be easily identified and it clear distinguishes the chatter prediction zones before the actual machining process. This in turn causes the improvement of the life of the cutting tool and makes estimation for the surface finish of the job. The cutting points operated above the boundary levels are treated as the unstable cutting zones while the below points are considered as the stable cutting points. In the present paper, the two directional cutting dynamics parameters are taken as $\mathrm{K}_{\mathrm{x}}=\mathrm{K}_{\mathrm{y}}=2.1 \times 10^{8} \mathrm{~N} / \mathrm{m}$, damping ratios in two directions as $\xi_{x}=\xi_{y}=.01$, diameter of the cutting tool as $15 \mathrm{~mm}$, helix angle of cutting tooth as $68^{0}$, cutting tooth as 4 , and the cutting pressure as $750 \mathrm{~N} / \mathrm{mm}^{2}$. With the frequency response values obtained from the Finite element modelling the stability lobe boundaries are plotted by considering down milling process with different depths of the radial immersions and are shown in the figure 4 . It is observed from these figures, as the percentage of the radial immersion increases there is an increase in the limit of axial depth of cut.
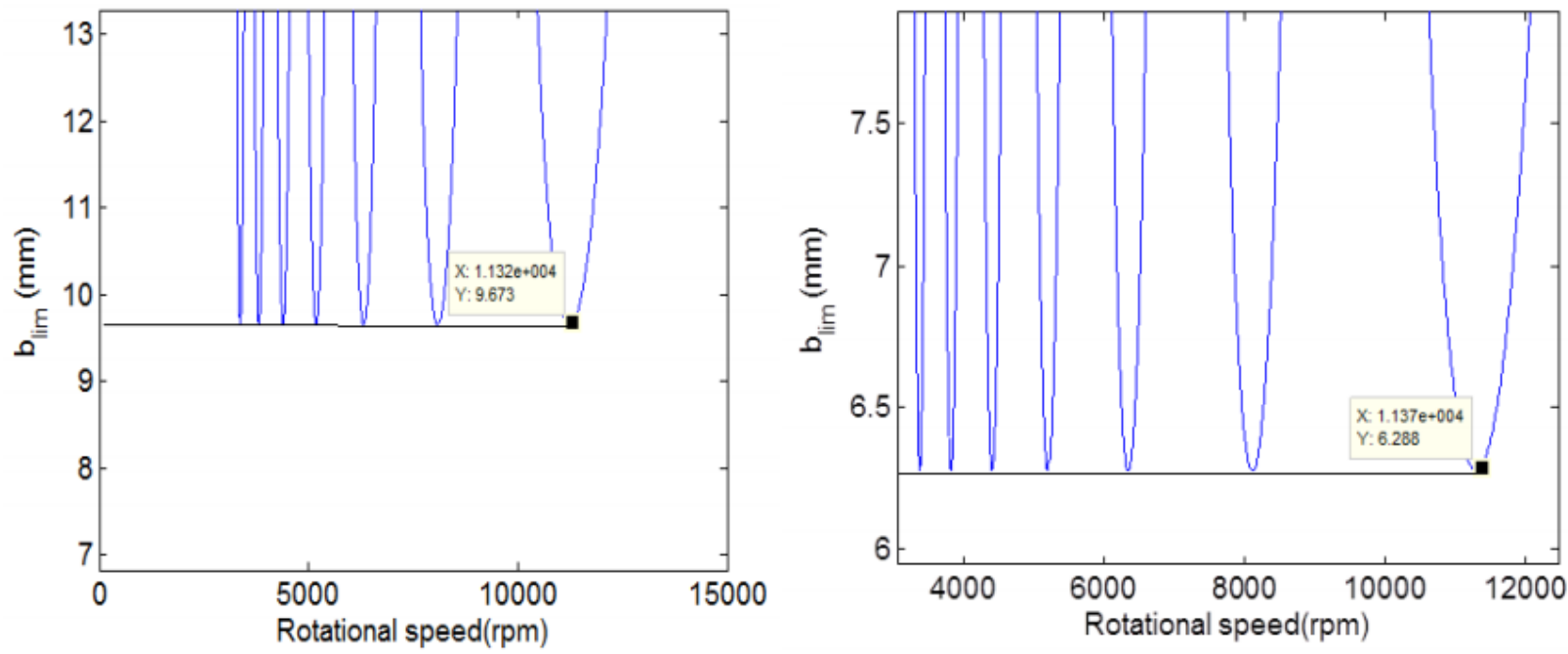

(a) 30\% Radial immersion

(b) $50 \%$ Radial immersion
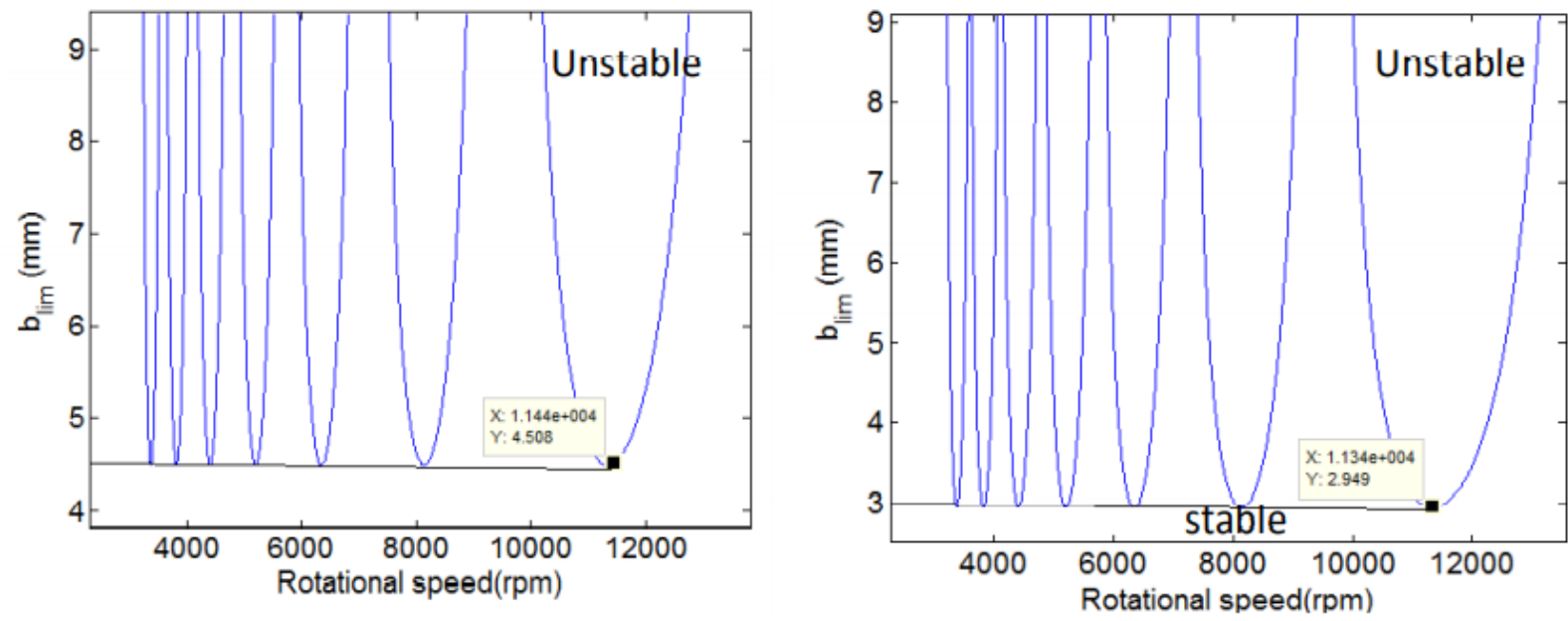

(c) $75 \%$ Radial immersion

(d) $100 \%$ Radial immersion

Fig. 4. Stability lobe plots for different percentages of radial immersion

\subsection{Prediction of dynamic stabilityfor the tool overhang and bearing span}

To develop an optimized spindle for the cutting process to reduce the effect the chatter vibration levels several process parameters such as high peak frequency at the tool tip, minimum weight of the integrated spindle tool system, maximum value of the dynamic stiffness, large depth of cut etc. are considered as objective parameters. Among these several geometric and cutting process parameters only few parameters like the 
dynamic stiffness of the cutting tool and the axial depth of cut are prime most factors that critically influence the chatter vibration levels during the machining process. The geometric design factors that critically influence the self excited vibrations are locations of the bearing, preload effect of the bearing, dimensions of the spindle, housing, overhang length of the cutting tool portion and helical angle of the cutting tool. In the present work, the design parameters are considered for the analysis such as tool overhang length and bearing span for the analysis. Figure 5 shows the effect of the overhang length of the cutting tool and the corresponding frequency responses are plotted for different lengths. It is observed that, when the length of the portion of the cutting tool is increased in the tool holder, there is change in the trend of the responses to the left of the frequency axis.
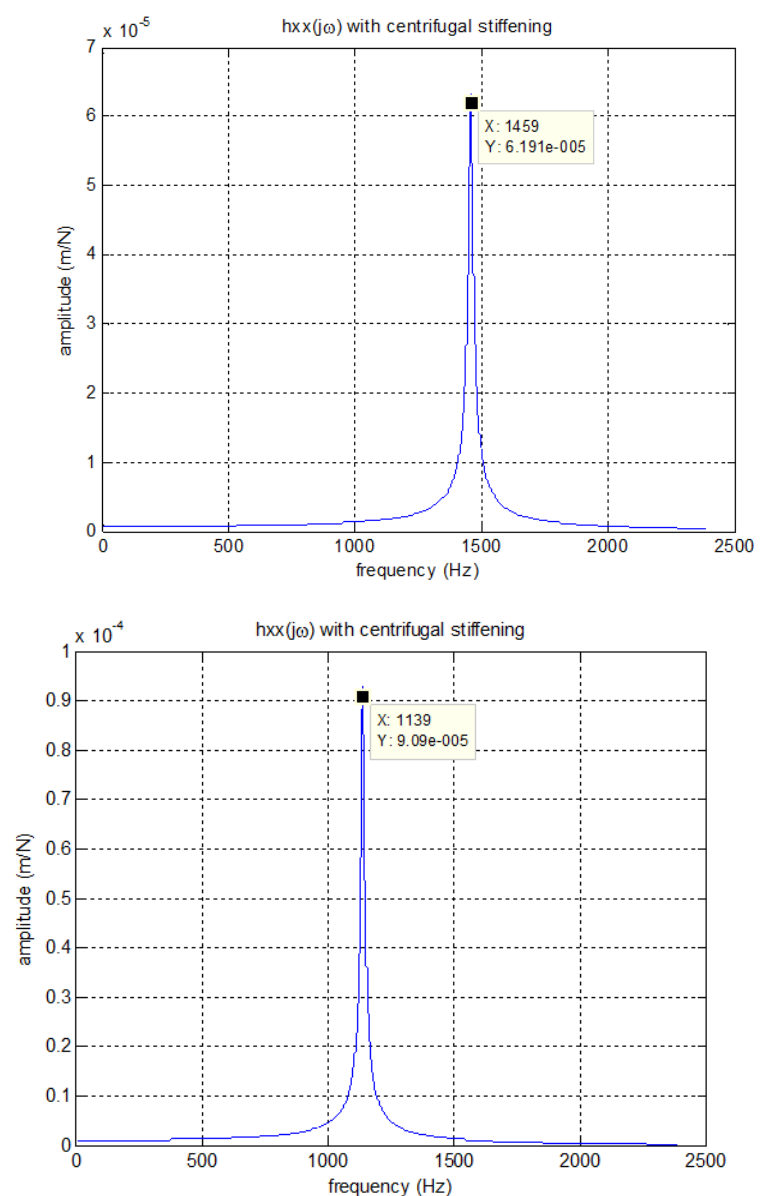

(a)FRF at $75 \mathrm{~mm}$ tool overhang

(b)FRF at $85 \mathrm{~mm}$ tool overhang

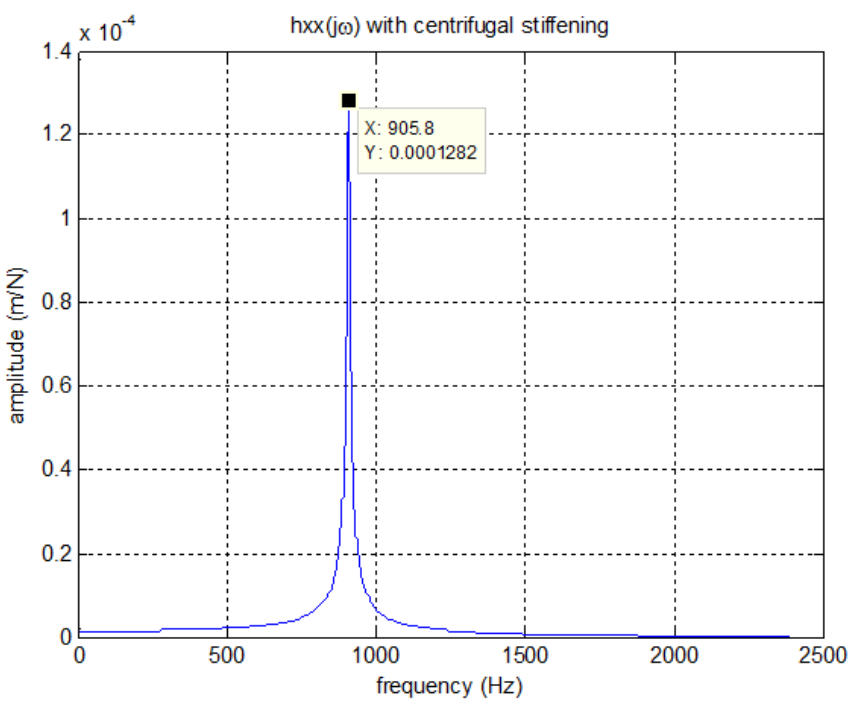

(c)FRF at $95 \mathrm{~mm}$ tool overhang

Figure 5. FRF at different bearing span ratios

Using the above tool tip frequency responses the stability lobes are plotted at different lengths of the tool 
overhang length and are shown in the figure 6. It is identified that, as the tool overhang length increases and there is a corresponding decrease in the level in the average stable depth of cut. It is confirmed that the first mode of natural frequency has a critical effect on both the spindle dynamics as well as on the machining dynamics of the cutting process.
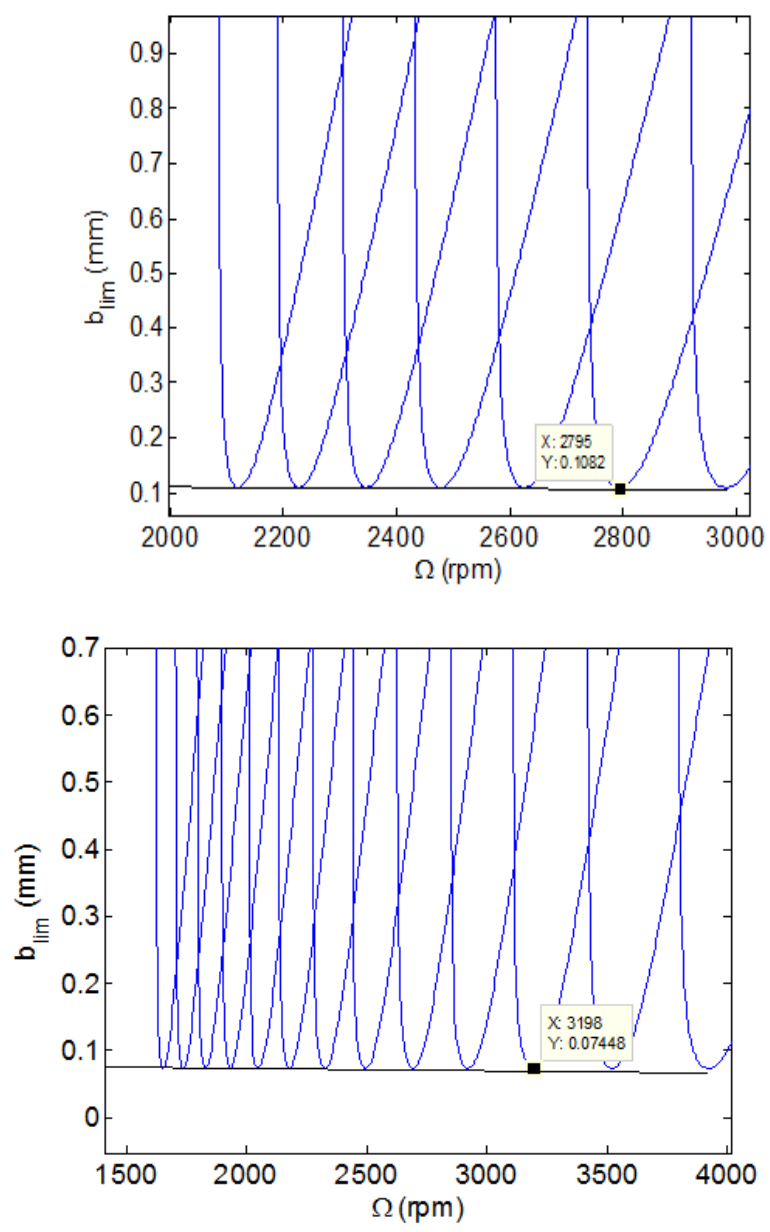

(a) $75 \mathrm{~mm}$ tool overhang

(b) $85 \mathrm{~mm}$ tool overhang

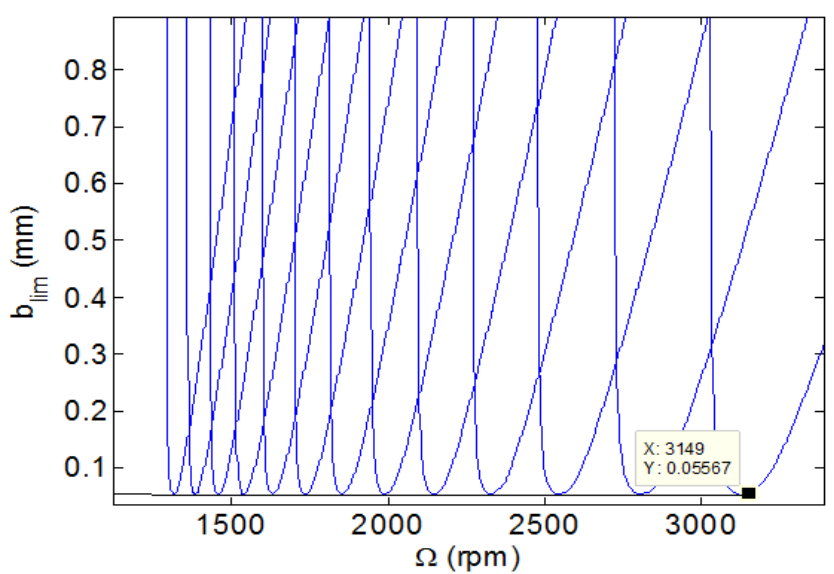

(c) $95 \mathrm{~mm}$ tool overhang

Fig. 6. Stability lobe diagrams for different lengths of tool overhang

Furthermore, the bearing span effect on the stability on the spindle tool system is evaluated for different spans and are shown in the figure.7. It is clearly evident from these simulations, as a small increment in the values of the bearing span, there is little change in the first mode of the natural frequency. Further, at a span value of $350 \mathrm{~mm}$ it is clearly identified that the second mode of vibraton plays a critical role and influences the 
dynamic stability of the spindle tool system and is shown in the figure 7(c)
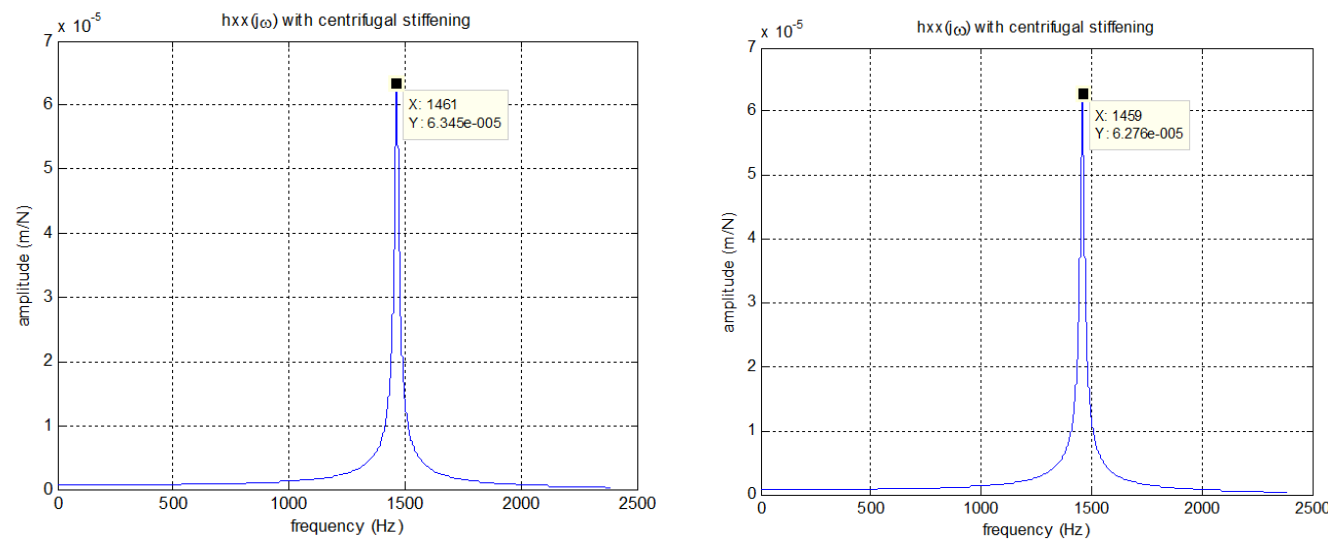

(a) $150 \mathrm{~mm}$ bearing span

(b) $250 \mathrm{~mm}$ bearing span

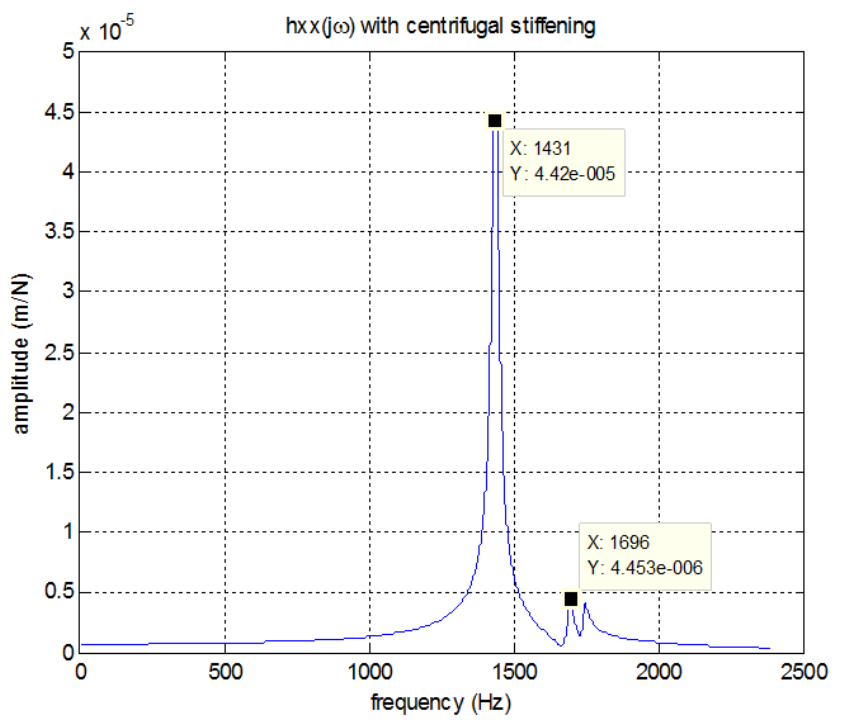

(c) $350 \mathrm{~mm}$ bearing span

Fig. 7. Tool tip frequency response at different bearing spans

The corresponding stability lobes were plotted with the above calculated real part of the frequency responses at the tool tip and are shown in the figure 8 . It is clearly observed that, at a bearing span value of $350 \mathrm{~mm}$, the secondary mode of frequency critically influences the machining stability and it forms the competing lobes or secondary lobes. Here the real part of the low frequency range is much more stiffer than the second mode of the frequency response. As the value of the bearing span length increases, there is a slight decrement in the values of the average stable depth of cut.

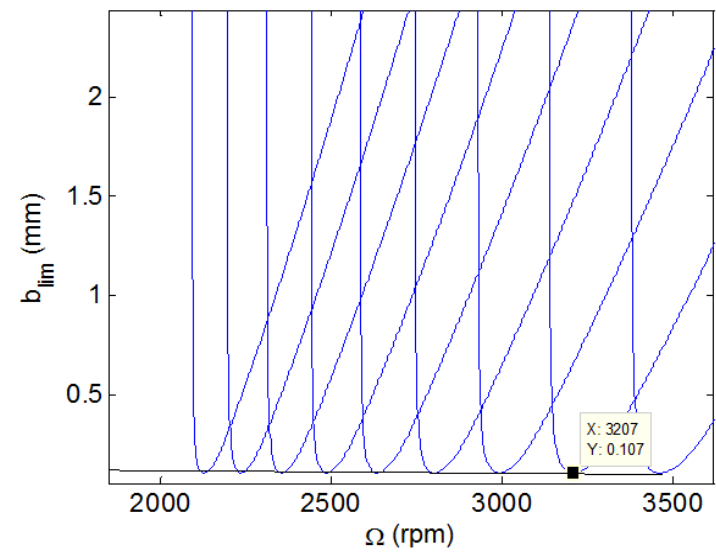

(a) $150 \mathrm{~mm}$ bearing span

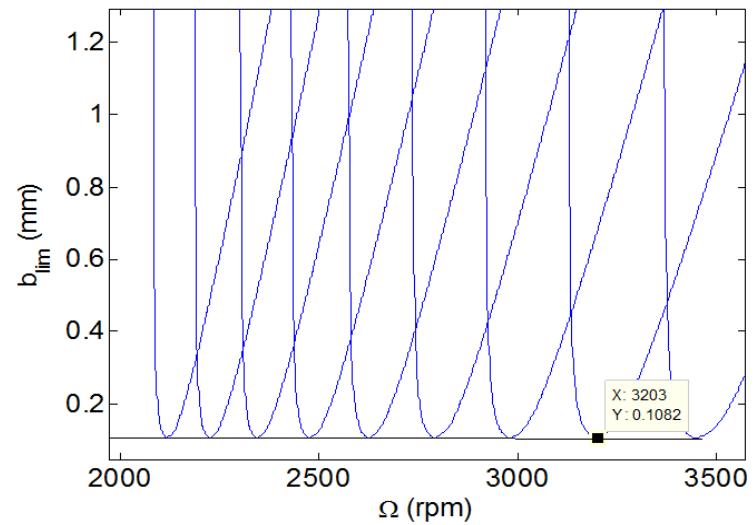

(b) $250 \mathrm{~mm}$ bearing span 


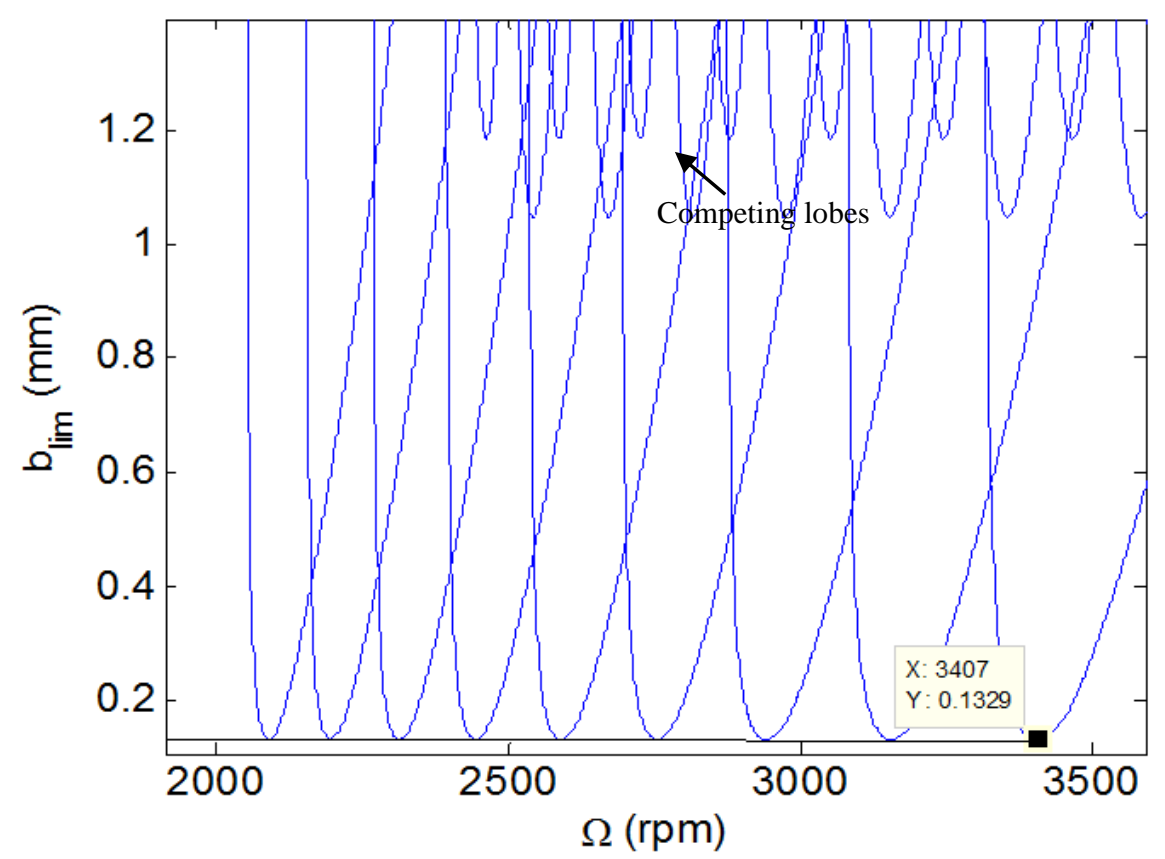

(c) $350 \mathrm{~mm}$ bearing span

Fig. 8. Stability lobe plots for different cases of bearing span

\subsection{Neural network optimization}

In the current work,the most extensively used approach,thefeedforwardbackpropagation neural networks,isdesignedforthepredictionof natural frequencies and axial depths of cut for theend-milling operation. The numerically simulated data are passed forward from the input to output layer, calculated errors are propagated back order to update the weights. From various trails, neural network (NN) skeleton is chosen as shown in figure 9.

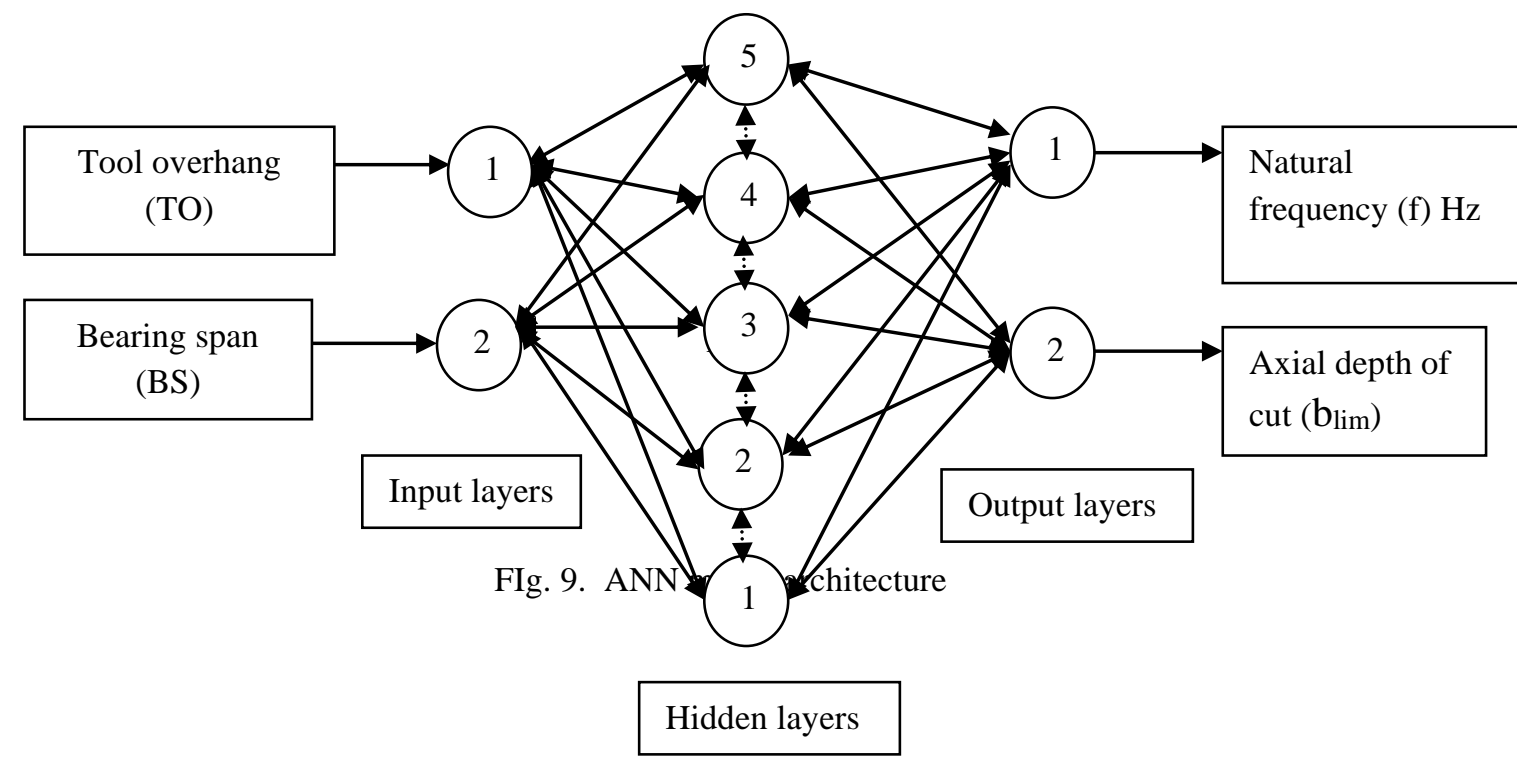

Here, the span between the two bearings and length of overhang portion of the cutting tool inside the tool holder are given as inputs and normalized values of fundamental frequencies and the average stable depths of cut are provided as target parameters. The natural frequencies (f) and average stable depths of cut $\left(b_{\text {lim }}\right)$ can be predicted for any unknown input data containing bearing span and tool overhang of the system. A code is written in MATLAB to train the network and to acquire the weights. Figure 10 shows the convergence of training trends with a five hidden neural architecture. 


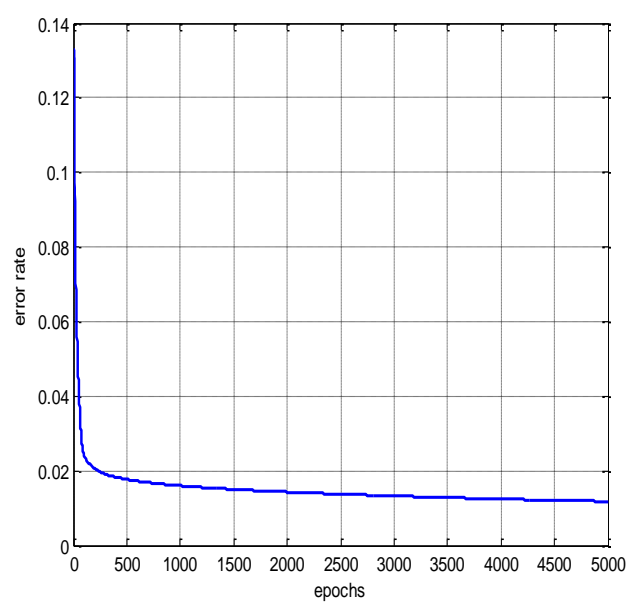

(a) convergence trend of (f )with TO

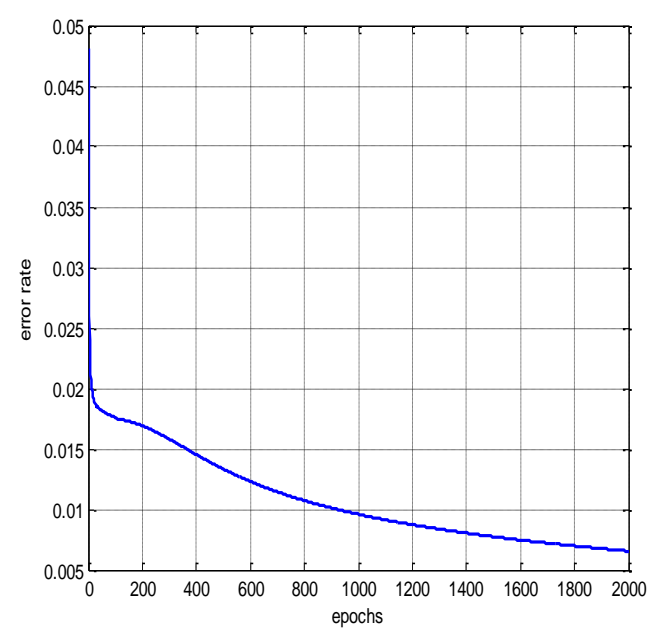

(c) convergence trend of (f) with BS

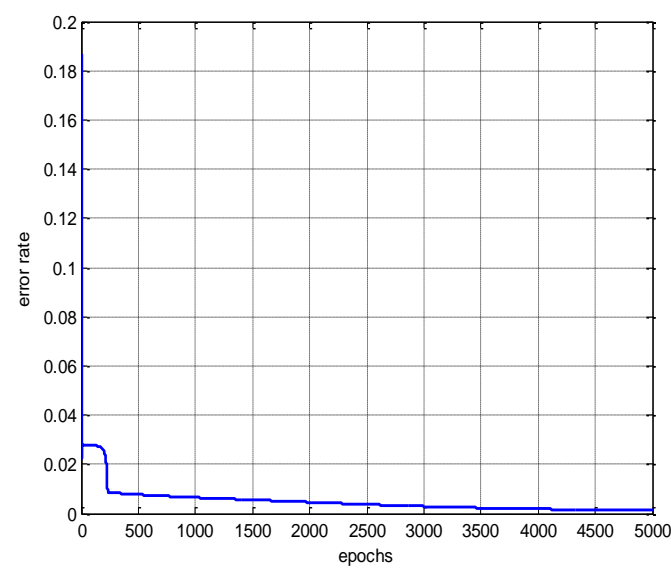

(b) convergence trend of $\left(\mathrm{b}_{\mathrm{lim}}\right)$ with $\mathrm{TO}$

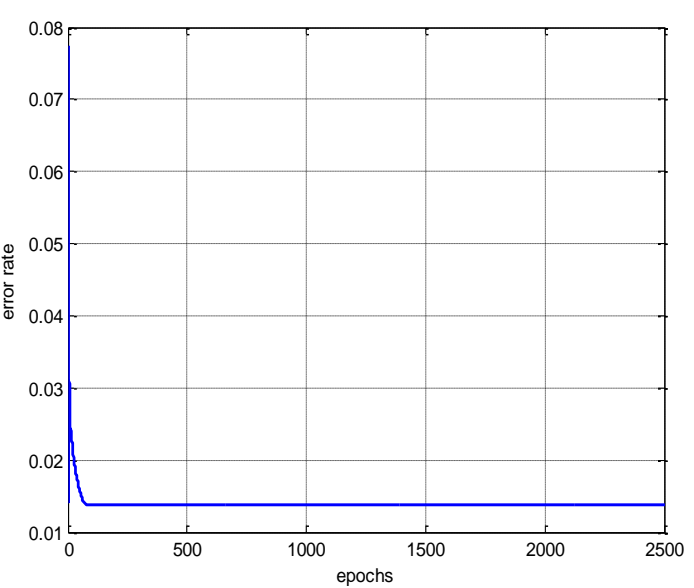

(b) convergence trend of $\left(\mathrm{b}_{\mathrm{lim}}\right)$ with BS

Figure. 10. Convergence trends of Tool overhang (TO) and Bearing span(BS)

The variation between the simulation data from the numerical simulations and neural network scheme is shown in the figure 11. As it is observed from these comparisons, the data for the overhang length of the tool(TO) and bearing span(BS) values are closer to each other, which confirm the accuracy of the numerical simulations.

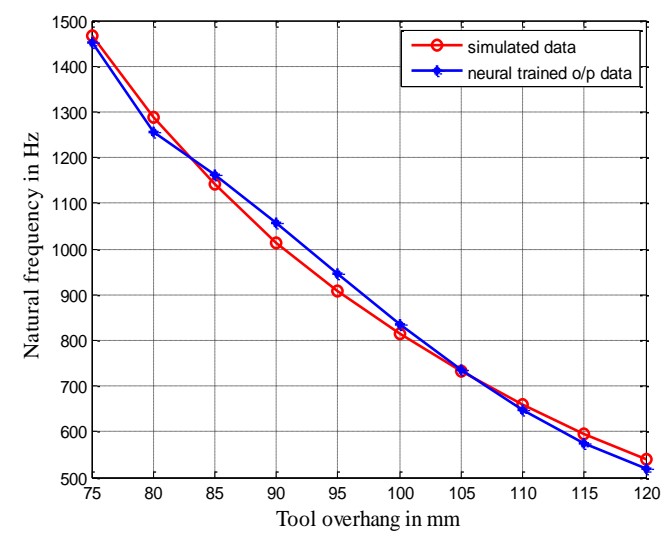

(a) Trained f with $\mathrm{T}$

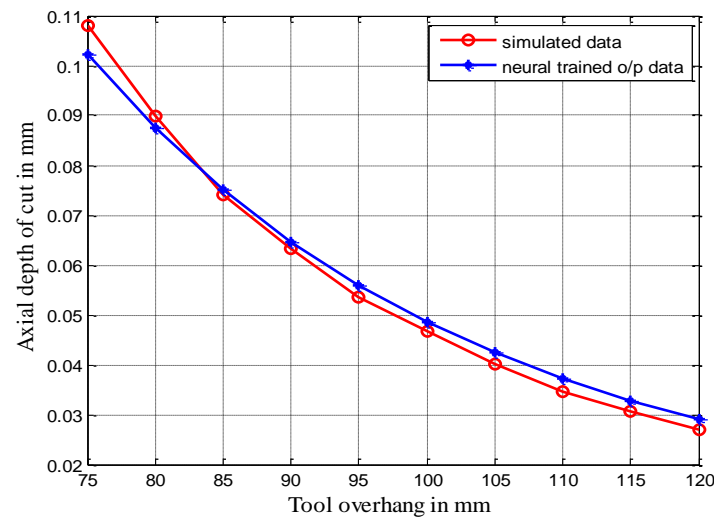

(b) Trained $b_{\text {lim }}$ with TO 

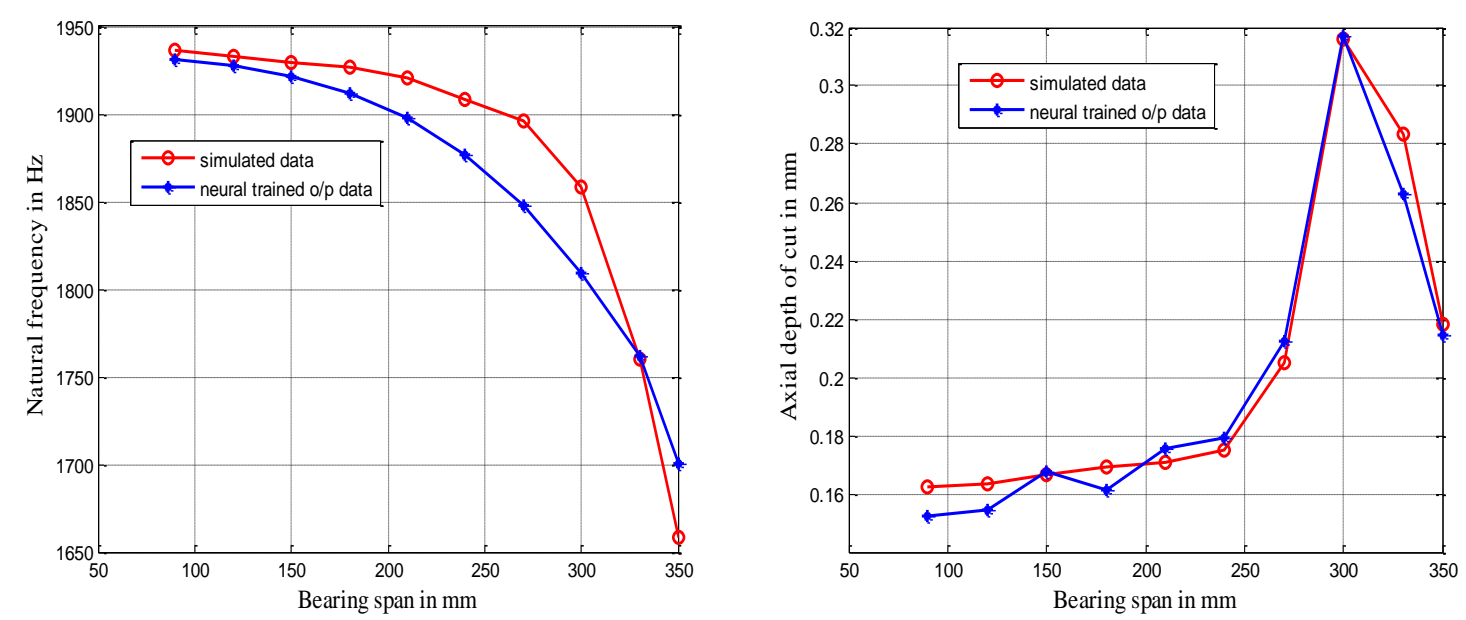

(c) Trained f with BS (d) Trained $b_{\text {lim }}$ with BS

Fig.11. Comparison of simulated data and neural trained output response

\section{Conclusions}

In the present paper a realistic data of the spindle tool system is considered for the numerical analysis with finite element approach by including the effects such a rotary inertia and shear deformation.

Fourier series method is employed to predict the stability lobes for end-milling process. Based on the formulation of dynamic milling with regeneration in the chip thickness, time varying directional factors and the interaction with the machine tool structure over a range of chatter frequencies the stability lobes are plotted for different depths of radial immersion in down milling process. From the results it is arrived that when machining aluminium as the depth of immersion increases the axial depth of cut decreases. For the practical machining of aluminium built-up edges will be formed on the cutting tool at high depth of cuts to avoid these effects a high percentage of radial immersion and high cutting speed is preferred which gives the stable machining. Further, the parametric design factors like span between the two bearings and overhang length of the tool of the spindle tool system is considered and their influence on the system dynamics are considered and the corresponding stability lobes are plotted. It is clearly identified from the analysis, as the span of bearing increases to a particular limit the second mode of frequency is critically influencing the system dynamics and further produce the competing lobes which causes the instability in the cutting process. An ANNscheme is implemented for the two design parametersand the results were generalized with the help of a feed-forward network, so as develop an observer system for the stability in cutting process.

\section{References:}

1. Altintas Y, Budak E (1995) Analytical prediction of stability lobes in milling. Annals of the CIRP 44: 357-362.

2. Tanga W.X, SongaQ.H (2009) Prediction of chatter stability in high-speed finishing end Milling considering multi-mode dynamics. Int. J. Machine Tools \&Manf209: 2585-2591.

3. GagnolaV, BouzgarrouB.C,Raya P, BarraC (2007) Model-based chatter stability predictionfor highspeed spindles.Int. J. Machine Tools \&Manf47:1176-1186.

4. BravoU,AltuzarraO,Lopez de Lacalle L.N, SanchezJ.A,CampaF.J (2005) Stability limits of milling considering the flexibility of the workpieceand the machine.Int. J. Machine Tools \&Manf45:1669-1680.

5. Suzuki N, Kurata Y, Kato T, Hino R, Shamoto E (2012) Identification of transfer function by inverse analysis of self-excited chattervibration in milling operations.Precision Engineering 36:568-575.

6. Lin C.W, TuJ.F (2007) Model-Based Design of Motorized SpindleSystems to Improve Dynamic Performanceat High Speeds. Journal of Manufacturing Process 9: 94-108.

7. CaoH,HolkupT,AltintasY (2011) A comparative study on the dynamics of high speed spindleswith respect to different preload mechanisms. Int. J. Machine Tools \& Manf 57:871-883.

8. S. Jiang and S. Zheng, (2010) Dynamic Design of a High-SpeedMotorized Spindle-Bearing System.Journal of Mechanical Design ASME,132, 0345011-0345015.

9. PengaZ.K, JacksonM.R, GuoL.Z, Parkin R.M, G. Meng (2010) Effects of bearing clearance on the chatter stability of milling process. Nonlinear Analysis: Real World Applications 11: 3577-3589.

10. Gao S.H, Meng G (2011) Research of the spindle over hang and bearing span on the system milling stability. Arch Appl Mech 81:1473-1486 
11. Ozturk E, Kumar U, Turner S, Schmitz T (2012) Investigation of spindle bearing preload on dynamics and stability limit in milling. Int. J. Machine Tools \&Manf 61:343-346.

12. RaphaelG. S, ReginaldoT.C, (2014) A Contribution to Improve the Accuracy of Chatter Prediction in Machine Tools Using the StabilityLobeDiagram.J. of ManfScienceand Engg, ASME136:021005-7

13. Zheng C.M, Junz W. J. J, Sung C.F, (2014) Analytical Prediction of the Critical Depth of Cut and Worst Spindle Speeds for Chatter in EndMilling. J. of ManfScienceand Engg, ASME136:011003-10.

14. LiuJ, Chen X, (2014) Dynamic design for motorized spindles based onanintegratedmodel. IntJAdvManufTechnol 71:1961-1974.

15. Liu D, Zhang H,Tao Z, Su Y (2011) Finite element analysis of high-speed motorized spindle based on ANSYS. The open Mechanical Engineering Journal 5:1-10.

16. Nelson H.D (1980) A finite rotating shaft element using Timoshenko beam theory. J.of machine design 102:793-803. 